\title{
Meningismus, CTCAE
}

National Cancer Institute

\section{Source}

National Cancer Institute. Meningismus, CT CAE. NCI Thesaurus. Code C143669.

A disorder characterized by neck stiffness, headache, and photophobia resulting from irritation of the cerebral meninges. 